\title{
Update on atmospheric neutrinos
}

\author{
M. C. Gonzalez-Garcia, ${ }^{1,2, *}$ H. Nunokawa, ${ }^{1, \dagger}$ O. L. G. Peres, ${ }^{1, \dagger}$ T. Stanev, ${ }^{3, \S}$ and J. W. F. Valle ${ }^{1, \|}$ \\ ${ }^{1}$ Departament de Física Teòrica, Instituto de Física Corpuscular-C.S.I.C., Universitat de València 46100 Burjassot, València, Spain ${ }^{\text {Il }}$ \\ ${ }^{2}$ Instituto de Física Teórica, Universidade Estadual Paulista, Rua Pamplona 145, 01405-900 São Paulo, Brazil \\ ${ }^{3}$ Bartol Research Institute, University of Delaware, Newark, Delaware 19716
}

(Received 21 January 1998; published 7 July 1998)

\begin{abstract}
We discuss the impact of recent experimental results on the determination of atmospheric neutrino oscillation parameters. We use all published results on atmospheric neutrinos, including the preliminary large statistics data of Super-Kamiokande. We reanalyze the data in terms of both $\nu_{\mu} \rightarrow \nu_{\tau}$ and $\nu_{\mu} \rightarrow \nu_{e}$ channels using new improved calculations of the atmospheric neutrino flux. We compare the sensitivity attained in atmospheric neutrino experiments with those of accelerator and reactor neutrino oscillation searches, including the recent $\mathrm{CHOOZ}$ experiment. We briefly comment on the implications of atmospheric neutrino data in relation to future searches for neutrino oscillations with long baselines, such as the K2K, MINOS, ICARUS, and NOE experiments. [S0556-2821(98)02215-2]
\end{abstract}

PACS number(s): 14.60.Pq, 13.15.+g, 95.85.Ry

\section{INTRODUCTION}

Atmospheric neutrinos are produced in cascades initiated by collisions of cosmic rays with the Earth's atmosphere [1]. Some of the mesons produced in these cascades, mostly pions and kaons, decay into electron and muon neutrinos and anti-neutrinos. The predicted absolute fluxes of neutrinos produced by cosmic-ray interactions in the atmosphere are uncertain at the $20 \%$ level. The ratios of neutrinos of different flavor are however expected to be accurate to better than $5 \%$. Since $\nu_{e}$ is produced mainly from the decay chain $\pi$ $\rightarrow \mu \nu_{\mu}$ followed by $\mu \rightarrow e \nu_{\mu} \nu_{e}$, one naively expects a 2:1 ratio of $\nu_{\mu}$ to $\nu_{e}$. In practice, however, the expected ratio of muon-like interactions to electron-like interactions in each experiment is more uncertain $[2,3]$.

Several experiments have observed atmospheric neutrino interactions. Two underground experiments, Kamiokande [4,5] and IMB [6], use water-Cherenkov detectors. These experiments have detected a ratio of $\nu_{\mu}$-induced events to $\nu_{e}$-induced events smaller than the expected one [3]. In particular Kamiokande has performed separate analyses for both sub-GeV neutrinos [4] and multi-GeV neutrinos [5], which show the same deficit. Although some of the experiments, such as Fréjus [7] and NUSEX [8], have not found evidence for this anomaly, and others, e.g. Soudan2, are not yet conclusive, the recent Super-Kamiokande data [9] provides strong support for an atmospheric muon neutrino deficit. This encourages us to reconsider the analysis of atmospheric neutrino data from the point of view of a neutrino oscillation interpretation. The recent improved data sample of Super-

\footnotetext{
*Email address: concha@evalvx.ific.uv.es

${ }^{\dagger}$ Present address: Instituto Fisica Gleb Wataghin, Universidade Estadual de Campinas, 13083-970 Campinas, SP, Brazil. Email address: nunokawa@ifi.unicamp.br

FEmail address: operes@ flamenco.ific.uv.es

${ }^{\S}$ Email address: stanev@ bartol.udel.edu

"Email address: valle@ flamenco.ific.uv.es

Ithttp://neutrinos.uv.es
}

Kamiokande now has better statistics than achieved in the whole Kamiokande phase.

We also include the new data of Soudan2 [10] in our analysis. These new data as well as the previous experimental results are summarized in Table I. Here $R_{\mu / e} / R_{\mu / e}^{M C}$ denotes the double ratio of experimental-to-expected ratio of muon-like to electron-like events. The expected ratio $R_{\mu / e}^{M C}$ is obtained by folding a prediction for the atmospheric neutrino flux with the properties of every individual detector through a Monte Carlo (MC) procedure.

Apart from studying the impact of the new data, our motivation for the present reanalysis of atmospheric neutrino data is theoretical. In this regard, we first of all include the results of a recent calculation of the atmospheric neutrino fluxes as a function of zenith angles [11], including the muon polarization effect [12]. Moreover, we develop an independent procedure for the comparison of results from different experiments. We demonstrate that our theoretical calculation of the energy distribution of the event rates is in good agreement with the MC expectations. The comparison of the experimental results presented below thus reflects the significance of the atmospheric neutrino anomaly and provides evidence for neutrino oscillations.

In this paper we analyze the impact of recent experimental results on atmospheric neutrinos from Super-Kamiokande and Soudan 2 on the determinations of atmospheric neutrino

TABLE I. Results from the atmospheric neutrino experiments.

\begin{tabular}{ll}
\hline \hline Experiment & \multicolumn{1}{c}{$R_{\mu / e} / R_{\mu / e}^{M C}$} \\
\hline Super-Kamiokande sub-GeV & $0.635 \pm 0.035 \pm 0.053$ \\
Super-Kamiokande multi-GeV & $0.604 \pm 0.065 \pm 0.065$ \\
Soudan2 & $0.61 \pm 0.14 \pm 0.07$ \\
IMB & $0.55 \pm 0.11$ \\
Kamiokande sub-GeV & $0.6 \pm 0.09$ \\
Kamiokande multi-GeV & $0.59 \pm 0.1$ \\
Fréjus & $1.06 \pm 0.23$ \\
Nusex & $0.96 \pm 0.3$ \\
\hline
\end{tabular}


oscillation parameters, both for the $\nu_{\mu} \rightarrow \nu_{\tau}$ and $\nu_{\mu} \rightarrow \nu_{e}$ channels. In so doing we take into account recent theoretical improvements in flux calculations and neutrino-nucleon cross sections. The new Super-Kamiokande data produce a downwards shift in the allowed $\left(\sin ^{2} 2 \theta, \Delta m^{2}\right)$ region, when compared with pre-Super-Kamiokande results. Nevertheless we show that the $\nu_{\mu} \rightarrow \nu_{e}$ oscillation hypothesis is barely consistent with the recent negative result of the $\mathrm{CHOOZ}$ reactor [13]. The sensitivity attained in atmospheric neutrino observations in the $\nu_{\mu} \rightarrow \nu_{\tau}$ channel is also compared with those of accelerator neutrino oscillation searches, for example at E776 and E531, as well as the present CHORUS [33] and NOMAD results [34] in addition to the future experiments being discussed at present.

\section{ATMOSPHERIC NEUTRINO FLUXES}

A contemporary calculation of the atmospheric neutrino fluxes consists of a Monte Carlo procedure that folds the measured energy spectra and chemical composition of the cosmic ray flux at the top of the atmosphere with the properties of hadronic interaction with the light atmospheric nuclei. Since the properties of the secondary mesons are extremely well known, the accuracy of the calculation is determined by the uncertainty of the two sets of assumptions - about the primary cosmic ray flux and about the hadronic interactions on light nuclei.

In order to avoid the uncertainty in the absolute magnitude of the cosmic ray flux experiments measure the ratio of electron to muon neutrinos, which is very stable in different calculations. The absolute normalization of the atmospheric neutrino flux is still very important for the interpretation of the observed muon neutrino deficit. If it turns out that the measured numbers of electron-like interactions agree with the predictions and there is an absolute deficit of muon-like interactions, the causes must be $\nu_{\mu}$ disappearance. If experiments measure the right amount of $\nu_{\mu}$ and an excessively large number of $\nu_{e}$ there must be a reason for $\nu_{e}$ appearance, such as $\nu_{\mu} \rightarrow \nu_{e}$ oscillations or a background process that generates $\nu_{e}$ or $e^{ \pm}$events in the detectors.

We use the new neutrino flux calculations of the Bartol group [11] which are performed with an updated version of the TARGET interaction event generator. The cosmic ray flux model is discussed in detail in Ref. [11]. The treatment of the hadronic collisions is very similar to that in earlier calculations [14]. There are only minor improvements in the treatment of the resonant region for low energy collisions and in the cross section for production of $\Lambda K$ pairs above $1000 \mathrm{GeV}$. The improvements in the low energy $(2-3 \mathrm{GeV})$ range slightly affects the fluxes of $100-300 \mathrm{MeV}$ neutrinos, while the kaon spectra at high energy change the neutrino to anti-neutrino ratios above $100 \mathrm{GeV}$ and thus mostly affect the predictions for the flux of upward going neutrino induced muons.

In the absence of geomagnetic effects the fluxes of $\mathrm{GeV}$ neutrinos are practically the same as those of Ref. [14]. A much more significant difference is introduced by the improved treatment of the geomagnetic effects [15]. The probability of low rigidity cosmic rays penetrating the atmo- sphere and producing neutrinos is calculated using a realistic model of the geomagnetic field, accounting for the shadow of the Earth. As a result the neutrino fluxes at experimental locations with a high geomagnetic cutoff, such as Kamioka, are significantly lower than in Ref. [14]. At high geomagnetic latitudes the new fluxes are comparable to the original ones.

This new set of fluxes, as well as the original one, belongs to a group (together with the calculation of Ref. [16]) of atmospheric neutrino flux predictions of relatively high magnitude. The expected magnitude of the atmospheric neutrinos was discussed by the authors of different predictions [17] who identified the reason for the differences in the treatment of the nuclear target effect in the hadronic collisions in the atmosphere. Calculations that assume that pion multiplicities in $p p$ and $p$ Air collisions are similar [18] predict low neutrino flux magnitudes. The event generator TARGET produces pion multiplicity that is higher by a factor of $\sim 1.6$ in $p A i r$ interactions above the resonant region.

The muon fluxes at different atmospheric depths generated with the same code as the new neutrino fluxes were compared to the measurements of the MASS experiment [19]. The predicted altitude profile of muons with energy above $1 \mathrm{GeV}$ agrees with the measured one extremely well.

\section{ATMOSPHERIC NEUTRINO CROSS SECTIONS AND EVENT DISTRIBUTIONS}

For each experiment the expected number of $\mu$-like and $e$-like events, $N_{\alpha}^{0}, \alpha=\mu, e$, in the absence of oscillations can be computed as

$$
\begin{aligned}
N_{\alpha}^{0}= & N_{t} T \int \frac{d^{2} \Phi_{\alpha}}{d E_{\nu} d\left(\cos \theta_{\nu}\right)} \kappa_{\alpha}\left(h, \cos \theta_{\nu}, E_{\nu}\right) \\
& \times \frac{d \sigma}{d E_{\alpha}} \varepsilon\left(E_{\alpha}\right) d E_{\nu} d E_{\alpha} d\left(\cos \theta_{\nu}\right) d h,
\end{aligned}
$$

where $N_{t}$ is the number of protons in target and $T$ is the exposure time. Here $E_{\nu}$ is the neutrino energy and $\Phi_{\alpha}$ is the flux of atmospheric neutrinos of type $\alpha=\mu, e ; E_{\alpha}$ is the final charged lepton energy and $\varepsilon\left(E_{\alpha}\right)$ is the detection efficiency for such a charged lepton; $\sigma$ is the neutrino-nucleon interaction cross section, $\nu N \rightarrow N^{\prime} \ell ; \theta_{\nu}$ is the angle between the vertical direction and neutrinos $\left(\cos \theta_{\nu}=1\right.$ corresponds to the downward direction). For some experiments, such as Fréjus, we also include neutral current events which are misidentified as charged current ones. In Eq. (1) $\kappa_{\alpha}$ is the distribution of $h$ which is the slant distance from the production point to the sea level for $\alpha$ type neutrinos with energy $E_{\nu}$ and a zenith angle $\theta_{\nu}$. We took the distribution from Ref. [20] which is normalized as

$$
\int \kappa_{\alpha}\left(h, \cos \theta_{\nu}, E_{\nu}\right) d h=1 .
$$

As discussed in Sec. II, the neutrino fluxes, in particular in the sub-GeV range, depend on the solar activity. In order 


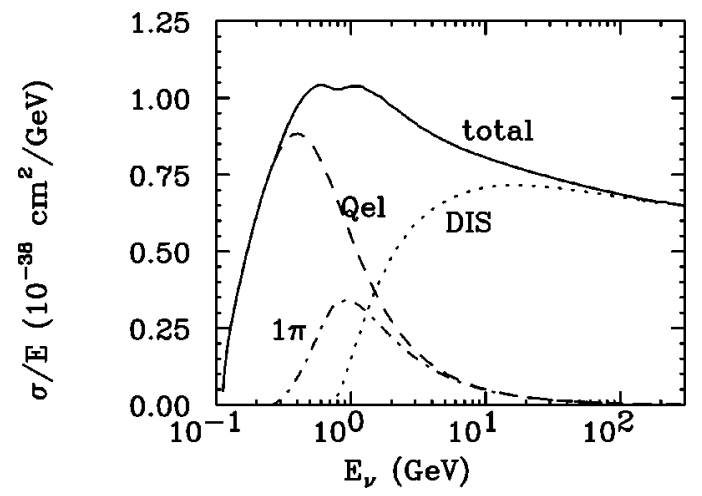

FIG. 1. Neutrino-nucleon cross sections used in this paper.

to take this fact into account we use in Eq. (1) and also in Sec. IV the averaged neutrino flux defined as follows:

$$
\Phi_{\alpha} \equiv c_{\max } \Phi_{\alpha}^{\max }+c_{\min } \Phi_{\alpha}^{\min }
$$

where $\Phi_{\alpha}^{\max }$ and $\Phi_{\alpha}^{\min }$ are the atmospheric neutrino fluxes when the sun is most active (solar maximum) and quiet (solar minimum), respectively. The coefficients $c_{\max }$ and $c_{\text {min }}$ $\left(=1-c_{\text {max }}\right)$ are determined according to the running period of each experiment assuming that the flux changes linearly with time between solar maximum and minimum. This is a first order correction for the solar modulation of the primary cosmic ray flux which has not been included in previous analyses.

\section{A. Cross sections}

In order to determine the expected event rates for the various experiments we use the neutrino-nucleon cross sections presented in Fig. 1. We consider separately the contributions to the cross section from the exclusive channels of lower multiplicity, quasi-elastic scattering and single pion production, and include all additional channels as part of the deep inelastic (DIS) cross section [21]:

$$
\sigma^{C C}=\sigma_{Q E}+\sigma_{1 \pi}+\sigma_{D I S}
$$

The quasi-elastic cross section for a neutrino with energy $E_{\nu}$ is given by [22]

$$
\begin{aligned}
\frac{d \sigma_{Q E}}{d\left|q^{2}\right|}\left(\nu n \rightarrow \ell^{-} p\right)= & \frac{M^{2} G_{F}^{2} \cos ^{2} \theta_{c}}{8 \pi E_{\nu}^{2}} \\
& \times\left[A_{1}\left(q^{2}\right)-A_{2}\left(q^{2}\right) \frac{s-u}{M^{2}}\right. \\
& \left.+A_{3}\left(q^{2}\right) \frac{(s-u)^{2}}{M^{4}}\right]
\end{aligned}
$$

where $s-u=4 M E_{\nu}+q^{2}-m_{\ell}^{2} ; M$ is the proton mass, $m_{\ell}$ is the charged lepton mass and $q^{2}$ is the momentum transfer. For $\nu p \rightarrow \ell^{+} n$, the same formula applies with the change $A_{2} \rightarrow-A_{2}$. The functions $A_{1}, A_{2}$, and $A_{3}$ can be written in terms of axial and vector form factors,

$$
\begin{aligned}
A_{1}= & \frac{m_{\ell}^{2}-q^{2}}{4 M^{2}}\left[\left(4-\frac{q^{2}}{M^{2}}\right)\left|F_{A}\right|^{2}-\left(4+\frac{q^{2}}{M^{2}}\right)\left|F_{V}^{1}\right|^{2}\right. \\
& -\frac{q^{2}}{M^{2}}\left|\xi F_{V}^{2}\right|^{2}\left(1+\frac{q^{2}}{4 M^{2}}\right)-\frac{4 q^{2}}{M^{2}} \operatorname{Re}\left(F_{V}^{1 \star} \xi F_{V}^{2}\right) \\
& \left.-\frac{m^{2}}{M^{2}}\left(\left|F_{V}^{1}+\xi F_{V}^{2}\right|^{2}+\left|F_{A}\right|^{2}\right)\right] \\
A_{2}= & -\frac{q^{2}}{M^{2}} \operatorname{Re}\left[F_{A}^{\star}\left(F_{V}^{1}+\xi F_{V}^{2}\right)\right] \\
A_{3}= & \frac{1}{4}\left(\left|F_{A}\right|^{2}+\left|F_{V}^{1}\right|^{2}-\frac{q^{2}}{4 M^{2}}\left|\xi F_{V}^{2}\right|^{2}\right),
\end{aligned}
$$

where we have neglected second order currents and we have assumed CVC (conservation of vector current). With this assumption all form factors are real and can be written as

$$
\begin{aligned}
F_{V}^{1}\left(q^{2}\right)= & \left(1-\frac{q^{2}}{4 M^{2}}\right)^{-1}\left(1-\frac{q^{2}}{M_{V}^{2}}\right)^{-2} \\
& \times\left[1-\frac{q^{2}}{4 M^{2}}\left(1+\mu_{p}-\mu_{n}\right)\right] \\
\xi F_{V}^{2}\left(q^{2}\right)= & \left(1-\frac{q^{2}}{4 M^{2}}\right)^{-1}\left(1-\frac{q^{2}}{M_{V}^{2}}\right)^{-2}\left(\mu_{n}-\mu_{p}\right) \\
F_{A}= & F_{A}(0)\left(1-\frac{q^{2}}{M_{A}^{2}}\right)^{-2} \cdot
\end{aligned}
$$

$\mu_{p}$ and $\mu_{n}$ are the proton and neutron anomalous magnetic moments and the vector mass, $M_{V}^{2}=0.71 \mathrm{GeV}^{2}$, is measured with high precision in electron scattering experiments. The largest uncertainties in this calculation are associated with the axial form factor. In our simulation we use $F_{A}(0)=$ -1.23 which is known from neutron beta decay. The axial mass used by the different collaborations varies in the range $M_{A}^{2}=0.71-1.06 \mathrm{GeV}^{2}$.

So far we have neglected nuclear effects. The most important of such effects is due to the Pauli principle. Following Ref. [22] we include it by using a simple Fermi gas model. In this approximation the cross section of a bound nucleon is equal to the cross section of a free nucleon multiplied by a factor $\left(1-N^{-1} D\right)$. For neutrons,

$$
\begin{aligned}
& D=Z \text { for } 2 z \leqslant u-v, \\
& D=\frac{1}{2} A\left[1-\frac{3 z}{4}\left(u^{2}+v^{2}\right)+\frac{z^{3}}{3}+\frac{3}{32 z}\left(u^{2}-v^{2}\right)^{2}\right] \\
& \quad \text { for } u-v \leqslant 2 z \leqslant u+v,
\end{aligned}
$$

$D=0$ for $2 z \geqslant u+v$

with $z=\left[\sqrt{\left(q^{2}+m_{\ell}^{2}\right)^{2} /\left(4 M^{2}\right)-q^{2}}\right] /\left(2 k_{f}^{2}\right), \quad u=(2 N / A)^{1 / 3}$, and $v=(2 Z / A)^{1 / 3}$. Here $A, Z, N$ are the nucleon, proton and neutron numbers and $k_{f}$ is the Fermi momentum, $k_{f}$ 

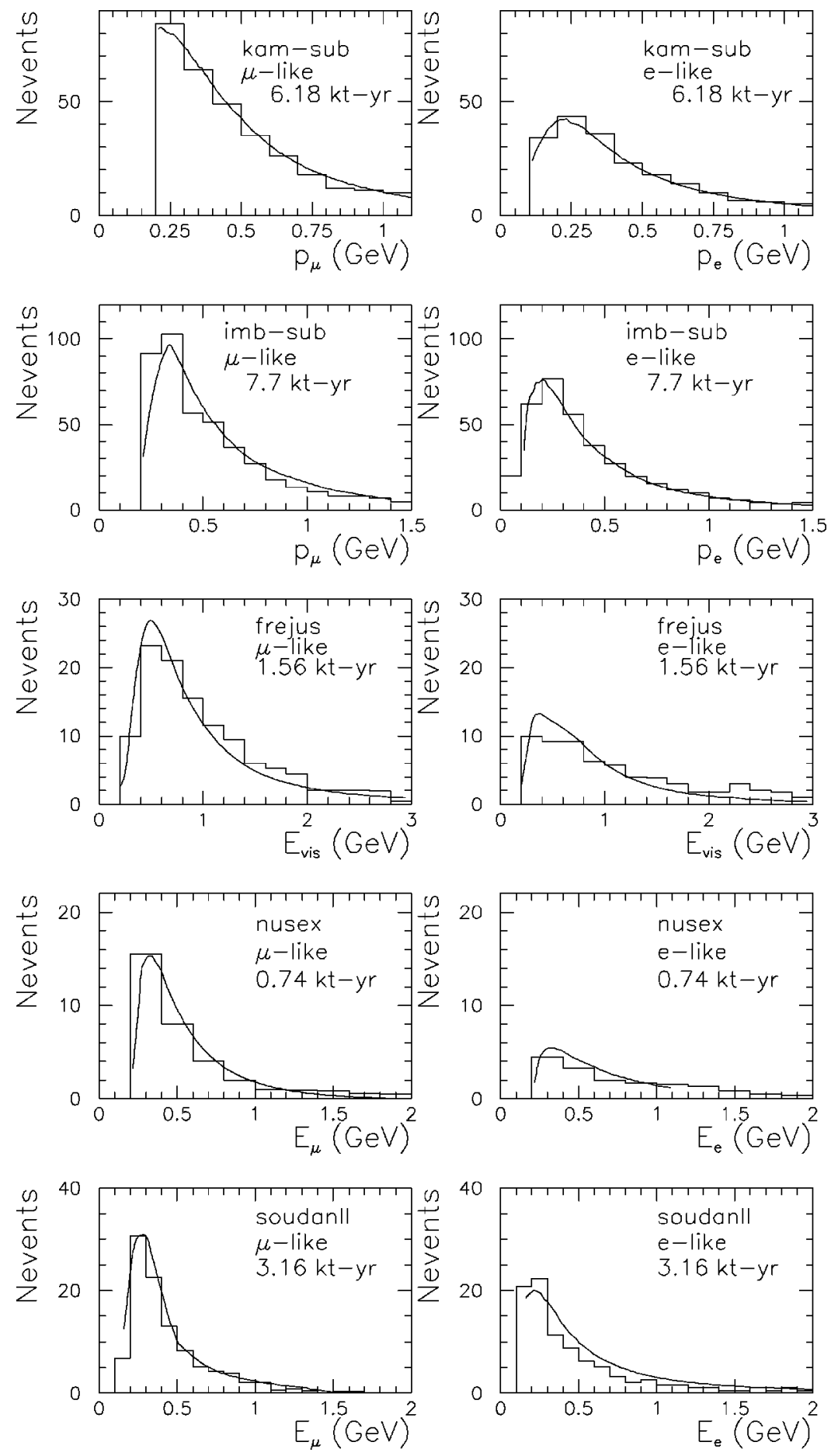

FIG. 2. The expected energy distribution of sub-GeV events (histogram) compared with our prediction (full line).

$=0.225(0.26)$, for oxygen (iron). For protons, the same formula applies with the exchange $N \leftrightarrow Z$. The effect of this factor is to decrease the cross section. The decrease is larger for a smaller neutrino energy. For energies above $1 \mathrm{GeV}$ the nuclear effects lead to an $8 \%$ decrease on the quasi-elastic cross section.

For a single pion production we use the model of Fogli and Nardulli [23] which includes hadronic masses below $W$ $=1.4 \mathrm{GeV}$. Deep inelastic cross sections are usually described in terms of the variables $y=1-E_{\ell} / E_{\nu}$ and $x=-q^{2} /\left(2 M E_{\nu} y\right)$. In the parton model

$$
\frac{d \sigma_{D I S}}{d x d y}\left(\begin{array}{c}
\nu \\
\bar{\nu}
\end{array}\right)=\frac{G_{F}^{2} s x}{4 \pi}\left[F_{1} \mp F_{3}+\left(F_{1} \pm F_{3}\right)(1-y)^{2}\right]
$$

where $F_{1}$ and $F_{3}$ are given in terms of the parton distributions. For isoscalar targets $F_{1}=2 \Sigma_{i}\left(q_{i}+\bar{q}_{i}\right)$ and $F_{3}=\Sigma_{i}\left(\bar{q}_{i}\right.$ $\left.-q_{i}\right)$. In order to avoid double counting we follow the approach of Ref. [21] and we integrate the deep inelastic con- 


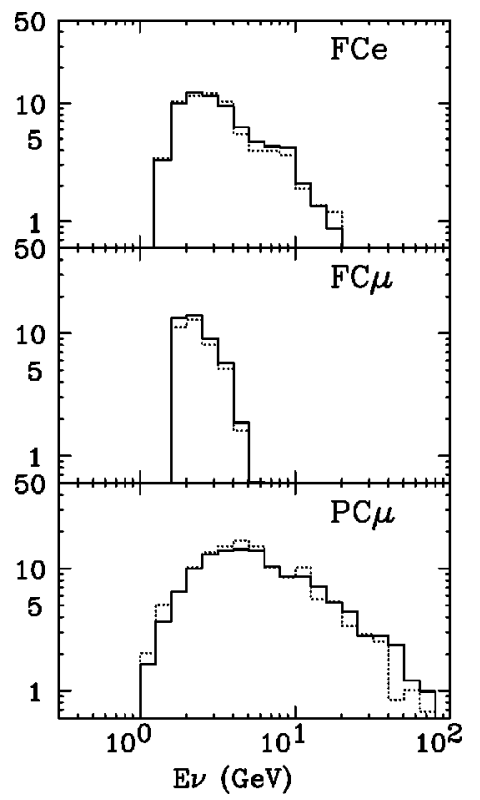

FIG. 3. The expected neutrino energy distribution of Kamiokande Multi-GeV events (dashed histogram) compared with our prediction (full histogram).

tribution in the region $W>W_{c}$ which implies $2 M E_{\nu} y(1$ $-x) \geqslant W_{c}^{2}-M^{2}$ where $W_{c}=1.4 \mathrm{GeV}$.

The final necessary ingredients are the detector efficiencies given by the experiments. These are, in general, functions of the incident neutrino energy and the detected lepton energy and flavor. We took these efficiencies from Refs. [25] for Kamiokande sub-GeV and IMB, [7] for Fréjus, and [8] for Nusex. The efficiencies for the Kamiokande multi-GeV and $e$-like events for Soudan 2 are provided by the experimentalists and for Soudan $2 \mu$-like events the efficiencies are determined in such a way that the energy distributions are well reproduced. For the Super-Kamiokande we are making some approximations based on the information also provided by the experimentalists, as discussed below.

\section{B. Event distributions}

In order to verify the quality of our simulation we compare our predictions for the energy distribution of the events with the Monte Carlo simulations of the different experiments in absence of oscillation. In Fig. 2 we show our predictions superimposed with those from the experimental Monte Carlo simulations for the sub-GeV experiments, Kamiokande sub-GeV [4], IMB [6], Frejus [7], Nusex [8] and Soudan2 [10]. We can see that the agreement is very good. No additional normalization of the event rates has been performed. Our results are in agreement with those of Ref. [24].

Similarly, in Fig. 3 we show the distribution of the fully contained electron-like events and fully and partially contained muon events for the Kamiokande multi-GeV sample compared with the experimental Monte Carlo prediction given in Fig. 2 of Ref. [5]. Some comments are necessary. In order to obtain these distributions we have used detailed experimental efficiencies of Kamiokande for detecting fully contained and partially contained electron and muon events
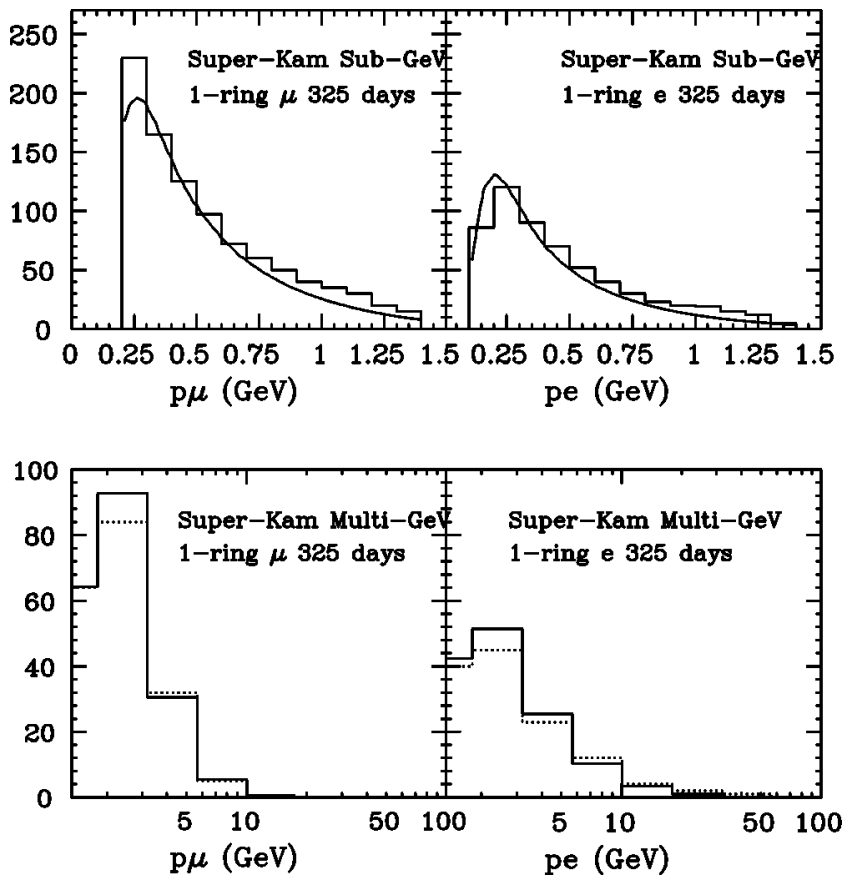

FIG. 4. The expected energy distribution of Super-Kamiokande events. For the sub-GeV events the histogram represents the $\mathrm{MC}$ expectation while the full line is our prediction. For the Multi-GeV events the full histogram is our result while the dashed histogram gives the $\mathrm{MC}$ prediction. Both our prediction and the $\mathrm{MC}$ prediction are based on the same flux calculations [11].

[25]. One must take into account that the Monte Carlo distributions given in Fig. 2 of Ref. [5] were generated using the fluxes of Honda et al. [16] while we used the fluxes of Gaisser et al. [14]. Thus we have an absolute prediction for the number of events for Kamiokande multi-GeV data and for their energy distribution which is obtained under the same assumptions for the cross sections and neutrino fluxes as the other sub-GeV experiments. For the sake of comparison we also show in Fig. 5 (upper two panels) the angular distribution of the events for Kamiokande multi-GeV data in the absence of oscillations as obtained from our calculation.

In Fig. 4 we also plot, as in Fig. 2, the expected energy distribution for Super-Kamiokande sub-GeV and multi-GeV data [9]. We also plot in Fig. 5 the angular distribution for Super-Kamiokande sub-GeV (middle two panels) and multi$\mathrm{GeV}$ data (lower two panels). We have used, as an approximation, the preliminary acceptances [26] of SuperKamiokande for 325.8 days for fully contained events and, 293 days for partially contained events as detection efficiencies for final leptons, for sub-GeV as well as multi-GeV data. In order to obtain the angular distribution of expected events for the multi-GeV range we have assumed that the lepton direction is the same as the incident neutrino direction. Actually for the Kamiokande multi-GeV data, the average angle between the incident neutrino and the lepton direction is about $15^{\circ}$. In our calculation we have simulated this difference by smearing the angular distribution with a Gaussian distribution with a one-sigma width of $15^{\circ}$. As seen in Fig. 5 the effect of this approximation is small. At this point it is worth noting that the angular distribution for multi-GeV 

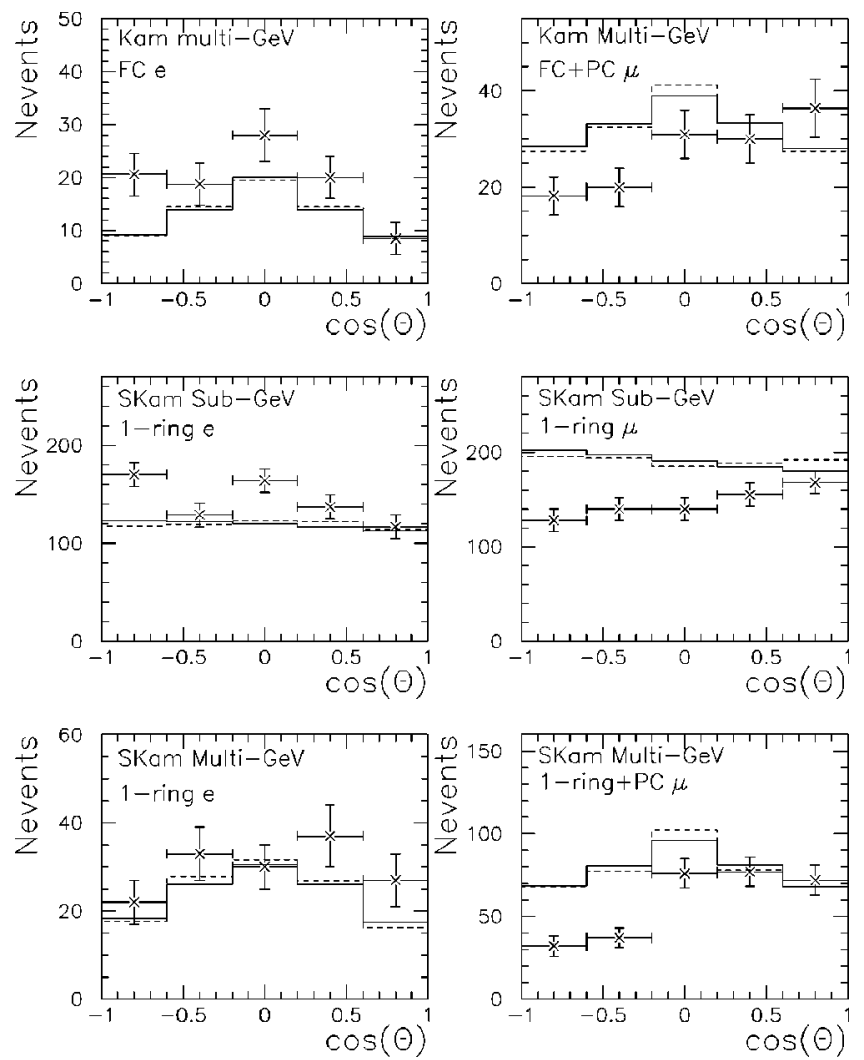

FIG. 5. The expected angular distribution of Kamiokande multi$\mathrm{GeV}$ events and Super-Kamiokande events (dashed histogram) obtained by Monte Carlo simulation by the experimental group compared with our predictions (full histogram) and the experimental data. We note that in these figures the MC prediction is based on Honda et al. fluxes [16] whereas ours is based on Bartol fluxes [11] normalized to the total number of expected events with the Honda MC fluxes.

electrons in the Super-Kamiokande sample is flatter than in the Kamiokande data. The main reason for this zenith-angle shape difference is because of the smaller selection efficiency for the 1-ring $e$-like events at high energy in the
super-Kamiokande analysis [25]. As a result the mean neutrino energy was shifted to a lower value and the mean angle between the incident neutrino and the lepton direction became larger. We have simulated this effect by increasing the one-sigma width of the smearing Gaussian to $25^{\circ}$ for the super-Kamiokande multi-GeV electrons which effectively flattens the angular distribution as seen in Fig. 5.

On the other hand, for events in the sub-GeV range we have carefully taken into account the difference between the incoming neutrino angle and the detected charged lepton scattering angle which is a function of the incoming neutrino energy. As can be seen in Fig. 5 this leads to a much flatter expected angular distribution for the sub-GeV neutrinos, in agreement with the prediction from the experimental MC results.

We also estimate the expected the ratio in the absence of oscillation as

$$
R_{\mu / e}^{0}=\frac{N_{\mu}^{0}}{N_{e}^{0}},
$$

where $N_{\mu}^{0}$ and $N_{e}^{0}$ are computed by Eq. (1). In Table II we present our prediction for the expected ratio in the absence of oscillations for the various experiments and compare it with the expected MC results [4-10]. Table II also displays our prediction for the expected ratio for the Kamiokande multi$\mathrm{GeV}$ and Super-Kamiokande zenith angle distribution. We see that the agreement between $R_{\mu / e}^{M C}$ and our prediction $R_{\mu / e}^{0}$ is very good for most of the experiments.

\section{ATMOSPHERIC NEUTRINO DATA FITS}

We now consider the simplest interpretation of the atmospheric neutrino anomaly in terms of the neutrino oscillation hypothesis. For definiteness we assume a two-flavor oscillation scenario, in which the $\nu_{\mu}$ oscillates into another flavor, either $\nu_{\mu} \rightarrow \nu_{e}$ or $\nu_{\mu} \rightarrow \nu_{\tau}$.

TABLE II. Our predictions for the ratio $\left(N_{\mu}^{0} / N_{e}^{0}\right)$ in the absence of oscillations compared to the MC expectations $\left(N_{\mu}^{M C} / N_{e}^{M C}\right)$ from each experimental group.

\begin{tabular}{|c|c|c|c|c|c|}
\hline & $\frac{N_{\mu}^{M C}}{N_{e}^{M C}}$ & $\frac{N_{\mu}^{0}}{N_{e}^{0}}$ & & $\frac{N_{\mu}^{M C}}{N_{e}^{M C}}$ & $\frac{N_{\mu}^{0}}{N_{e}^{0}}$ \\
\hline Fréjus & 1.9 & 1.8 & Super-Kamiokande (sub-GeV) & 1.6 & 1.6 \\
\hline Kamiokande (sub-GeV) & 1.55 & 1.6 & Bin1 & 1.7 & 1.6 \\
\hline IMB & 1.1 & 1.1 & $\operatorname{Bin} 2$ & 1.6 & 1.5 \\
\hline Soudan 2 & 1.05 & 1.1 & Bin3 & 1.5 & 1.5 \\
\hline Nusex & 1.9 & 1.8 & Bin4 & 1.5 & 1.6 \\
\hline Kamiokande (multi-GeV) & 2.3 & 2.4 & Bin5 & 1.7 & 1.5 \\
\hline Bin1 & 3.1 & 3.1 & Super-Kamiokande (multi-GeV) & 3.2 & 3.0 \\
\hline Bin2 & 2.4 & 2.4 & Bin1 & 3.8 & 3.4 \\
\hline Bin3 & 2.1 & 2.0 & Bin2 & 2.8 & 2.8 \\
\hline Bin 4 & 2.4 & 2.4 & Bin3 & 3.2 & 2.8 \\
\hline \multirow[t]{2}{*}{ Bin5 } & 3.2 & 3.2 & Bin4 & 2.9 & 2.8 \\
\hline & & & Bin5 & 4.2 & 3.5 \\
\hline
\end{tabular}




\section{A. Data analysis procedure}

In the presence of two-flavor neutrino oscillations, the expected number of $\mu$ and $e$-like events, $N_{\alpha}, \alpha=\mu, e$ is given by

$$
N_{\mu}=N_{\mu \mu}^{0}\left\langle P_{\mu \mu}\right\rangle+N_{e \mu}^{0}\left\langle P_{e \mu}\right\rangle, \quad N_{e}=N_{e e}^{0}\left\langle P_{e e}\right\rangle+N_{\mu e}^{0}\left\langle P_{\mu e}\right\rangle,
$$

where

$$
\begin{aligned}
N_{\alpha \beta}^{0}= & N_{t} T \int \frac{d^{2} \Phi_{\alpha}}{d E_{\nu} d\left(\cos \theta_{\nu}\right)} \kappa_{\beta}\left(h, \cos \theta_{\nu}, E_{\nu}\right) \\
& \times \frac{d \sigma}{d E_{\beta}} \varepsilon\left(E_{\beta}\right) d E_{\nu} d E_{\beta} d\left(\cos \theta_{\nu}\right) d h
\end{aligned}
$$

and

$$
\left\langle P_{\alpha \beta}\right\rangle=\frac{N_{t} T}{N_{\alpha \beta}^{0}} \int \frac{d^{2} \Phi_{\alpha}}{d E_{\nu} d\left(\cos \theta_{\nu}\right)} \kappa_{\beta}\left(h, \cos \theta_{\nu}, E_{\nu}\right) P_{\alpha \beta} \frac{d \sigma}{d E_{\beta}} \varepsilon\left(E_{\beta}\right) d E_{\nu} d E_{\beta} d\left(\cos \theta_{\nu}\right) d h
$$

Here $P_{\alpha \beta}$ is the oscillation probability of $\nu_{\beta} \rightarrow \nu_{\alpha}$ for given values of $E_{\nu_{\beta}}, \quad \cos \theta_{\nu} \quad$ and $h$, i.e., $P_{\alpha \beta} \equiv P\left(\nu_{\beta}\right.$ $\left.\rightarrow \nu_{\alpha} ; E_{\nu_{\beta}}, \cos \theta_{\nu}, h\right)$.

We note that for the $\nu_{\mu} \rightarrow \nu_{e}$ channel, Earth matter effects lead to oscillation probabilities which are different for neutrinos and anti-neutrinos. Therefore, we separately compute $P_{\alpha \beta}$ in Eq. (13) in order to correctly estimate the expected number of events in each experiment (see Sec. IV B for more discussion).

When combining the results of the experiments we do not make use of the double ratio, $R_{\mu / e} / R_{\mu / e}^{M C}$, but instead we treat the $e$ and $\mu$-like data separately, taking carefully into account the correlation of errors. Following Ref. [27] we define $\chi^{2}$ as

$$
\begin{aligned}
\chi^{2} \equiv & \sum_{I, J}\left(N_{I}^{\text {data }}-N_{I}^{\text {theory }}\right) \cdot\left(\sigma_{\text {data }}^{2}\right. \\
& \left.+\sigma_{\text {theory }}^{2}\right)_{I J}^{-1} \cdot\left(N_{J}^{\text {data }}-N_{J}^{\text {theory }}\right),
\end{aligned}
$$

where $I$ and $J$ stand for any combination of experimental data set and type of events considered, i.e, $I=(A, \alpha)$ and $J$ $=(B, \beta)$ where, $A, B=$ Fréjus, Kamiokande sub-GeV, $\mathrm{IMB}, \ldots$ and $\alpha, \beta=e, \mu$. In Eq. (14) $N_{I}^{\text {theory }}$ is the predicted number of events calculated by Eq. (11) whereas $N_{I}^{\text {data }}$ is the number of observed events. In Eq. (14) $\sigma_{\text {data }}^{2}$ and $\sigma_{\text {theory }}^{2}$ are the error matrices containing the experimental errors and the MC errors respectively. They can be written as

$$
\sigma_{I J}^{2} \equiv \sigma_{\alpha}(A) \rho_{\alpha \beta}(A, B) \sigma_{\beta}(B),
$$

where $\rho_{\alpha \beta}(A, B)$ stands for the correlation between the $\alpha$ like events in the $A$-type experiment and $\beta$-like events in the $B$-type experiment, whereas $\sigma_{\alpha}(A)$ and $\sigma_{\beta}(B)$ are the errors for the number of $\alpha$ and $\beta$-like events in $A$ and $B$ experiments, respectively. The dimension of the error matrix varies depending on the combination of experiments included in the analysis. For each individual experiment, the error matrix has a dimension of $2 \times 2$ whereas for the full experimental data set with binning (20 data for each flavor) its dimension is $40 \times 40$.
With this procedure of separately treating the $e$-like and $\mu$-like data with the correlation of errors, we avoid the nonGaussian nature of the double ratio, as pointed out in Ref. [27].

We compute $\rho_{\alpha \beta}(A, B)$ as in Ref. [27]. A detailed discussion of the errors and correlations used in our analysis can be found in the Appendix. In Table III we show the values of $\chi^{2}$ and the confidence level in the absence of oscillation. In our analysis, we have conservatively assumed a $30 \%$ uncertainty regarding the absolute neutrino flux, in order to generously account for the spread of neutrino flux predictions in different calculations. ${ }^{1}$

Next we minimize the $\chi^{2}$ function in Eq. (14) and determine the allowed region in the $\sin ^{2} 2 \theta-\Delta m^{2}$ plane, for a given confidence level, defined as

$$
\chi^{2} \equiv \chi_{\min }^{2}+4.61(9.21) \quad \text { for } 90(99) \% \text { C.L. }
$$

\section{B. $\nu_{\mu} \rightarrow \nu_{e}$ channel}

The results of our $\chi^{2}$ fit of atmospheric neutrino data obtained at the various individual water-Cerenkov and iron calorimeter detectors for the $\nu_{\mu} \rightarrow \nu_{e}$ channel are shown in Fig. 6. The allowed regions for each experiment lie to the right of the corresponding labeled line, except for the negative Frejus and Nusex experiments which are marked with the left-pointing arrows in the figure. So far we have not included in the above analysis the constraints that arise from the inclusion of the angular dependence of the data in the Kamiokande multi-GeV data as well as the SuperKamiokande data. In the right hand panel of Fig. 6 we show how the binned results of Kamiokande and SuperKamiokande give rise to a region of oscillation parameters that cuts out the large $\Delta m^{2}$ values. Moreover one can see that the Super-Kamiokande binned sub-GeV data yield a somewhat lower value of $\Delta m^{2}$ than the multi-GeV data.

\footnotetext{
${ }^{1}$ For a brief discussion of the effect of the assumed flux uncertainties, see Sec. V.
} 
TABLE III. Values of $\chi^{2}$ and confidence levels for each experiment in the absence of oscillations. For unbinned data the number of degrees of freedom is 2 while for combined binned data it is 10 .

\begin{tabular}{lcc}
\hline \hline Experiment & $\chi^{2}$ & C.L. $(\%)$ \\
\hline Fréjus & 0.56 & 24.4 \\
IMB & 8.4 & 98.5 \\
Soudan2 & 5.7 & 94.2 \\
Nusex & 0.39 & 17.7 \\
Kamiokande sub-GeV & 12.5 & 99.8 \\
Kamiokande multi-GeV unbinned & 8.7 & 98.7 \\
Kamiokande multi-GeV binned & 18.2 & 94.8 \\
Super-Kamiokande sub-GeV unbinned & 21.5 & 99.7 \\
Super-Kamiokande sub-GeV binned & 27.2 & 100.0 \\
Super-Kamiokande multi-GeV unbinned & 10. & 99.3 \\
Super-Kamiokande multi-GeV binned & 27.9 & 99.8 \\
\hline \hline
\end{tabular}

The effects of combining all atmospheric neutrino data from the various experiments for the $\nu_{\mu} \rightarrow \nu_{e}$ channel are shown in Fig. 7. This figure show the allowed $\nu_{\mu} \rightarrow \nu_{e}$ oscillation parameters for all experiments combined at 90 and 99\% C.L. For comparison we have also plotted in Fig. 7 the presently excluded region from reactor experiments, Krasnoyarsk [30], Bugey [31] and the recent CHOOZ long-baseline result [13].

We have so far neglected Earth matter effects [28], both in Fig. 6 and Fig. 7. In order to take into account the earth matter effect in our analysis we have separately computed, by numerical integration, the oscillation probabilities, $P\left(\nu_{\mu}\right.$ $\left.\rightarrow \nu_{e}\right)=P\left(\nu_{e} \rightarrow \nu_{\mu}\right)$ and $P\left(\bar{\nu}_{\mu} \rightarrow \bar{\nu}_{e}\right)=P\left(\bar{\nu}_{e} \rightarrow \bar{\nu}_{\mu}\right)$. This is necessary, since the matter effect distinguishes neutrinos from anti-neutrinos. We have used the approximate analytic expression for the electron density profile in the Earth obtained in Ref. [29]. In order to save computation (CPU) time we have neglected the matter effect for neutrino oscillation parameters in the range

$$
\frac{\Delta m^{2}}{E}>10^{-11} \mathrm{eV},
$$
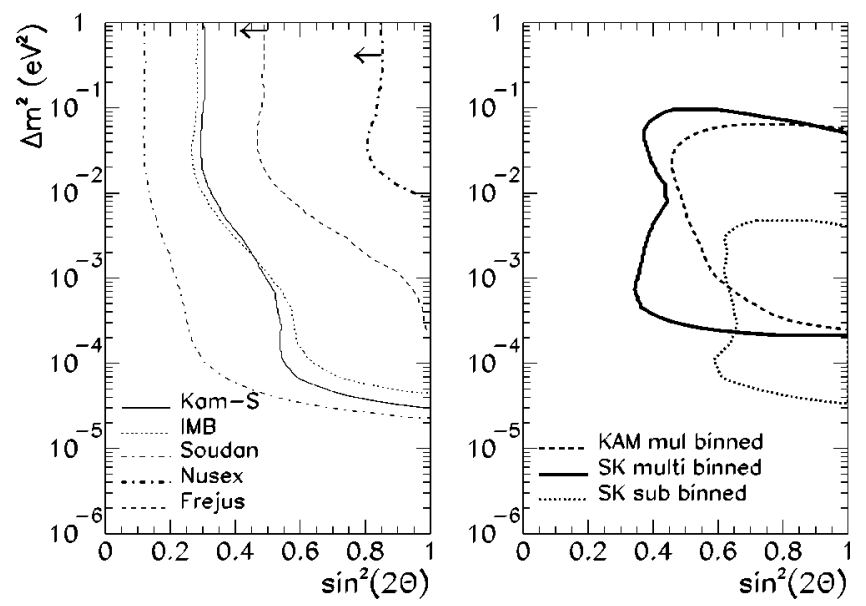

FIG. 6. The allowed $\nu_{\mu} \rightarrow \nu_{e}$ oscillation parameters at 90\% C.L. for each individual experiment neglecting Earth matter effects.

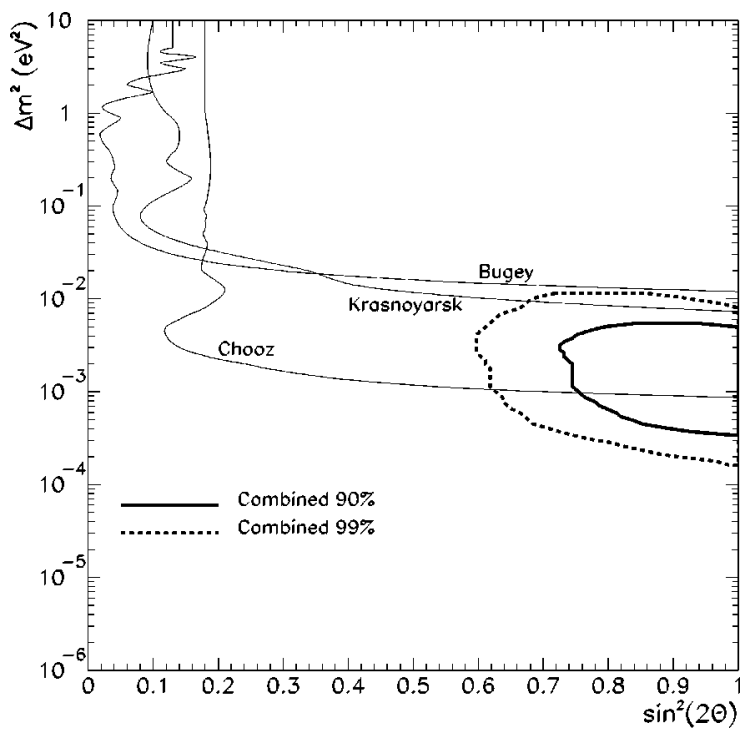

FIG. 7. The allowed $\nu_{\mu} \rightarrow \nu_{e}$ oscillation parameters for all experiments combined at 90 and $99 \%$ C.L. neglecting Earth matter effects. For a comparison we also plot the presently excluded region from reactor experiments.

since the maximum value of the matter potential (at the Earth center) is, at most,

$$
V_{\text {matter }} \sim 10^{-12} \mathrm{eV}
$$

and the matter effect on the probability is small if condition Eq. (17) is satisfied.

In Fig. 8 we show the allowed $\nu_{\mu} \rightarrow \nu_{e}$ oscillation parameters for each individual experiment including Earth matter effects. As in Fig. 6 the allowed regions for each experiment lie to the right of the corresponding labeled line, except for the negative Fréjus and Nusex experiments which are marked with the left-pointing arrows in the figure. Unlike the previous case where matter effects were neglected, a noticeable new feature in this case is that the Super-Kamiokande multi-GeV data now allows large $\Delta m^{2}$ values, even if binning is taken into account. The allowed $\nu_{\mu} \rightarrow \nu_{e}$ oscillation parameters for the Super-Kamiokande binned data combined at 90 and $99 \%$ C.L. including Earth matter effects in shown in Fig. 9. An interesting feature to note here is that by adding the matter effects the allowed regions lie higher in $\Delta m^{2}$ than when matter effects are neglected. This is because for smaller $\Delta m^{2}$, i.e. when $\Delta m^{2} \cos 2 \theta / 2 E$ is much smaller than $V_{\text {matter }}$, the effective conversion amplitude $\sin ^{2} 2 \theta_{m}$ where $\theta_{m}$ is the mixing angle in matter, is smaller than that of the vacuum one, i.e. $\sin ^{2} 2 \theta$. In other words, in this region matter suppresses the conversion and it becomes harder to fit for the atmospheric neutrino anomaly.

The allowed $\nu_{\mu} \rightarrow \nu_{e}$ oscillation parameters for all experiments combined at 90 and $99 \%$ C.L. including Earth matter effects is shown in Fig. 10. Again one can see that by adding the matter effects the allowed regions lift higher in $\Delta m^{2}$ than when matter effects are neglected. We found the best fit point at $\left(\sin ^{2} 2 \theta, \Delta m^{2}\right) \sim\left(0.97,2.6 \times 10^{-3} \mathrm{eV}^{2}\right)$ where $\chi_{\text {min }}^{2}$ $=62.7$ for 40 degrees of freedom. We would like to point out 

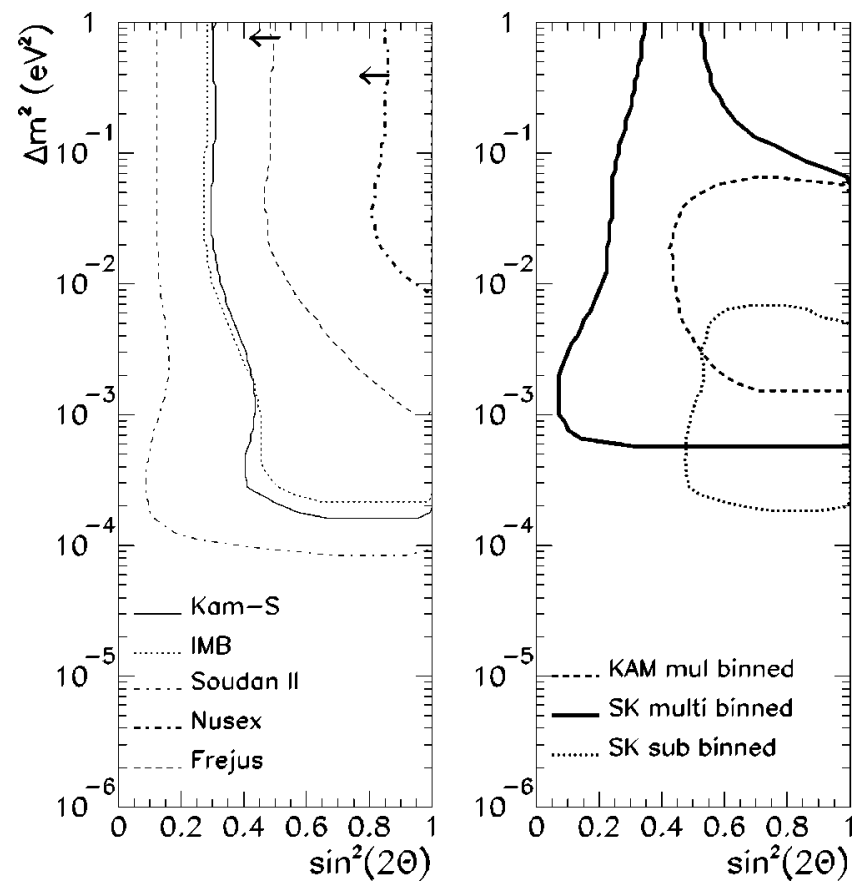

FIG. 8. The allowed $\nu_{\mu} \rightarrow \nu_{e}$ oscillation parameters at 90\% C.L. for each individual experiment including Earth matter effects.

that at this stage the weight of the experiments with negative results (NUSEX and Fréjus) is small enough not to modify the $\chi^{2}$ per degree of freedom $\left(\chi_{\min }^{2}=54.2\right.$ for 36 degrees of freedom when these experiments are removed).

It is instructive at this stage to compare the region determined by the atmospheric neutrino data fit with the presently excluded region from reactor experiments [31]. The inclusion of the matter effects becomes especially relevant when one makes a comparison with the long baseline reactor neutrino data, such as the recent data of $\mathrm{CHOOZ}$ [13]. One sees

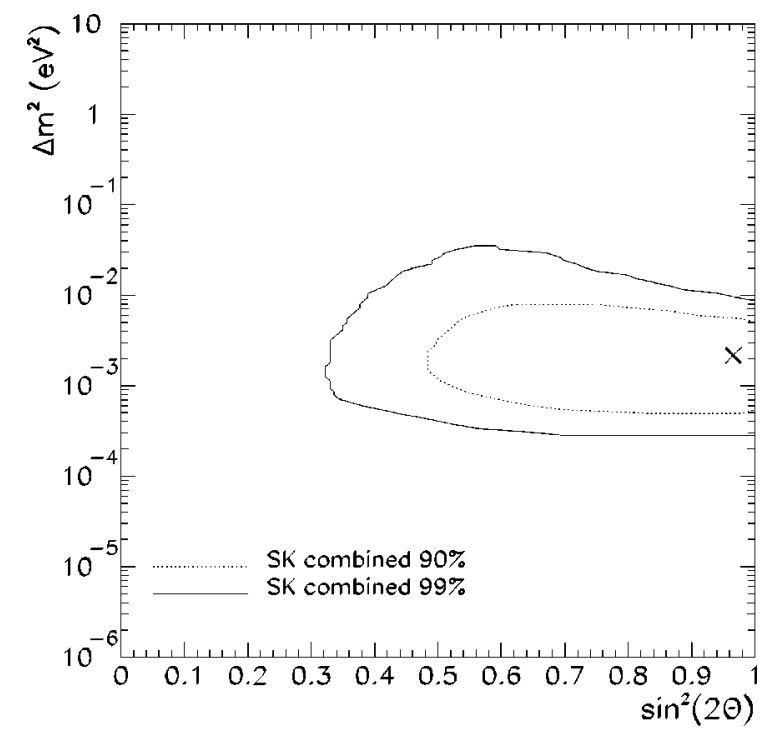

FIG. 9. The allowed $\nu_{\mu} \rightarrow \nu_{e}$ oscillation parameters for the Superkamiokande experiment combined at 90 and $99 \%$ C.L. including Earth matter effects. The cross represents the best fit point.

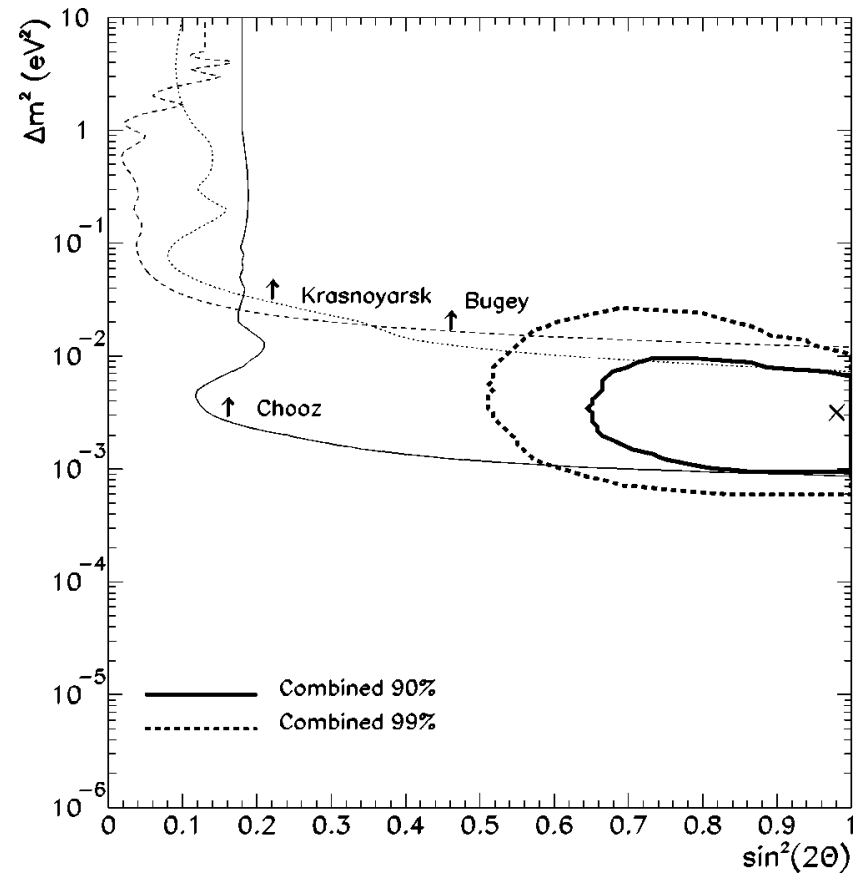

FIG. 10. The allowed $\nu_{\mu} \rightarrow \nu_{e}$ oscillation parameters for all experiments combined at 90 (solid) and 99\% C.L. (dashed) including Earth matter effects. For a comparison we also plot the presently excluded region from reactor experiments. The cross represents the best fit point.

that at $90 \%$ C.L. the $\nu_{\mu}$ to $\nu_{e}$ oscillation channel is ruled out as a solution of the atmospheric neutrino anomaly.

\section{C. $\nu_{\mu} \rightarrow \nu_{\tau}$ channel}

The results of our $\chi^{2}$ fit of atmospheric neutrino data obtained from the data of individual experiments for the $\nu_{\mu}$ $\rightarrow \nu_{\tau}$ channel are shown in Fig. 11. The allowed regions for each experiment lie to the right of the corresponding labeled line, except for the negative Fréjus and Nusex experiments. In the left part in Fig. 11 we have not included the constraints that arise from the inclusion of the angular depen-
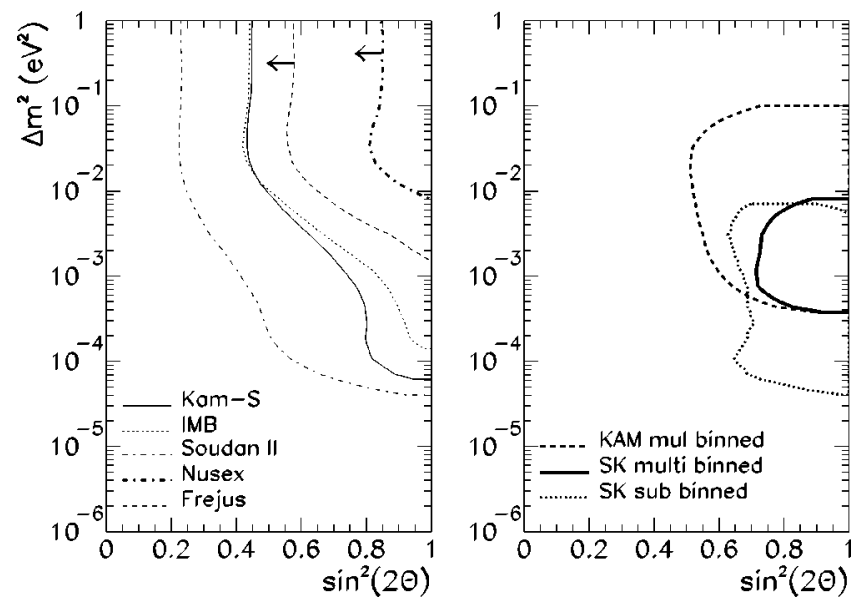

FIG. 11. The allowed $\nu_{\mu} \rightarrow \nu_{\tau}$ oscillation parameters at $90 \%$ C.L. for each individual experiment. 


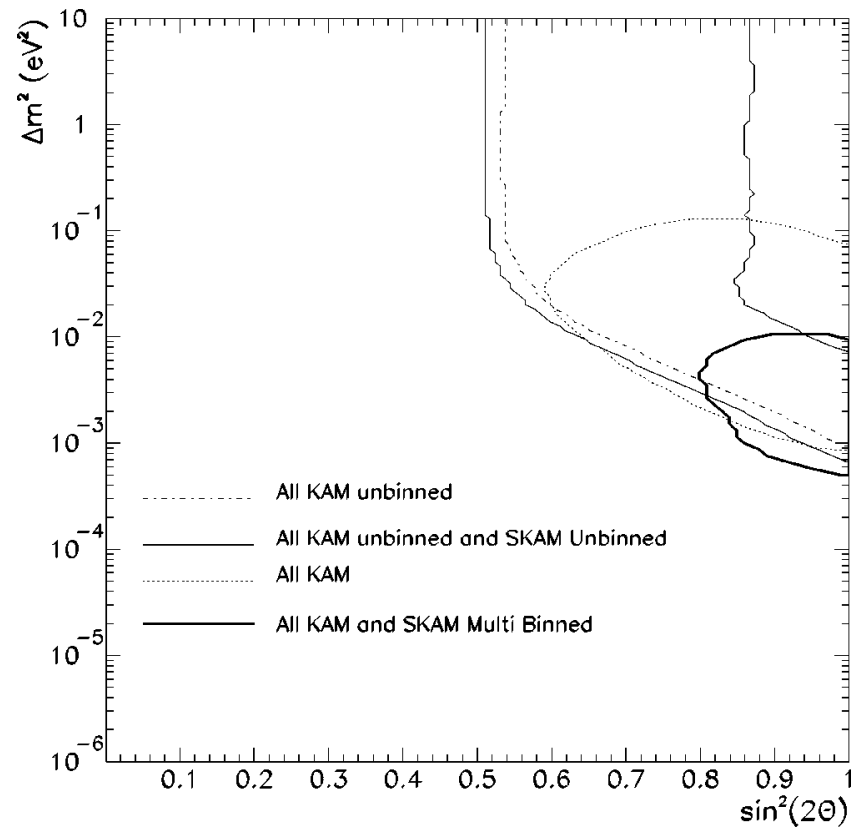

FIG. 12. The allowed $\nu_{\mu} \rightarrow \nu_{\tau}$ oscillation parameters at $90 \%$ C.L. for Kamiokande and Kamiokande plus Superkamiokande combined.

dence of the data in the Kamiokande multi-GeV data as well as in the Super-Kamiokande data.

It is instructive to compare the results obtained for the Kamiokande data with those obtained by including the recent Super-Kamiokande data. In Fig. 12 we show the allowed $\nu_{\mu} \rightarrow \nu_{\tau}$ oscillation parameters for Kamiokande and Kamiokande plus Super-Kamiokande combined. Some features are worth remarking. For example, the inclusion of the unbinned Super-Kamiokande data to the corresponding Kamiokande data leads to the exclusion of large mixing in the large $\Delta m^{2}$ region. On the other hand the inclusion of SuperKamiokande binned data leads to a substantially smaller region obtained from the Kamiokande full data sample, reflecting a real improvement.

In Fig. 13 we give the allowed $\nu_{\mu} \rightarrow \nu_{\tau}$ oscillation parameters for Super-Kamiokande combined at 90 and 99\% C.L. while in Fig. 14 we display the allowed $\nu_{\mu} \rightarrow \nu_{\tau}$ oscillation parameters for all experiments combined at 90 and $99 \%$ C.L. By comparing Fig. 13 with Fig. 14 one can see the weight of the Super-Kamiokande data sample in the total data sample collected by all experiments. We find that the best fit points lie at $\left(\sin ^{2} 2 \theta, \Delta m^{2}\right) \sim\left(1,1.3 \times 10^{-3} \mathrm{eV}^{2}\right)$ with $\chi_{\min }^{2}=14.4$ for the 20 degrees of freedom, for Super-Kamiokande only and $\left(\sin ^{2} 2 \theta, \Delta m^{2}\right) \sim\left(1,1.2 \times 10^{-3} \mathrm{eV}^{2}\right)$ with $\chi_{\min }^{2}=66.6$ for the 40 degrees of freedom, for all combined. The global fit to all experiments is still slightly better for the $\nu_{\mu} \rightarrow \nu_{e}$ channel. However, the difference between the quality of the fit for both channels is smaller now than in the pre-SuperKamiokande era, due to the angular distribution of SuperKamiokande multi-GeV data which strongly favors the $\nu_{\mu}$ $\rightarrow \nu_{\tau}$ channel.

The result of including the information on the zenith angle distribution of the events in the $\nu_{\mu}$ to $\nu_{\tau}$ fit is clearly to

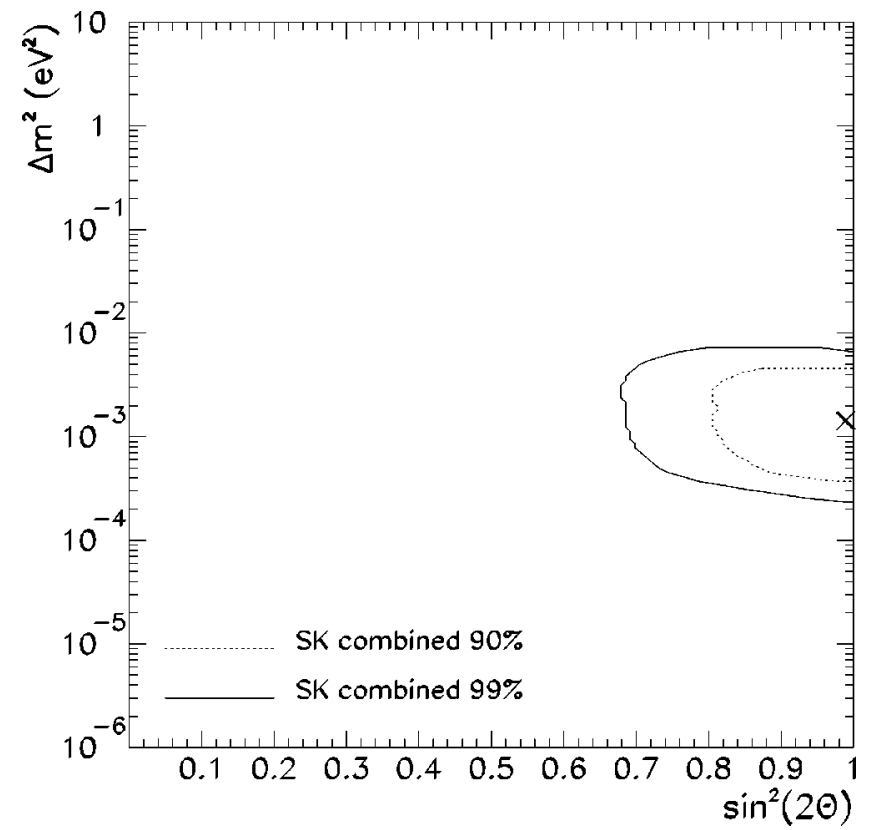

FIG. 13. The allowed $\nu_{\mu} \rightarrow \nu_{\tau}$ oscillation parameters for $\mathrm{Su}-$ perkamiokande combined at 90 and $99 \%$ C.L. The cross represents the best fit point.

cut the large values of $\Delta m^{2}$, as can be seen in all figures, namely Fig. 11, Fig. 12, Fig. 13 and Fig. 14.

One point worth noting is that the inclusion of the new Super-Kamiokande data produces a downward shift in the $\left(\sin ^{2} 2 \theta, \Delta m^{2}\right)$ region, when compared with pre-SuperKamiokande fits. The importance of the information obtained from the analysis of the atmospheric neutrino data analysis in relation to the results from accelerator experiments such as E776 and E531 and CDHSW [32], as well as

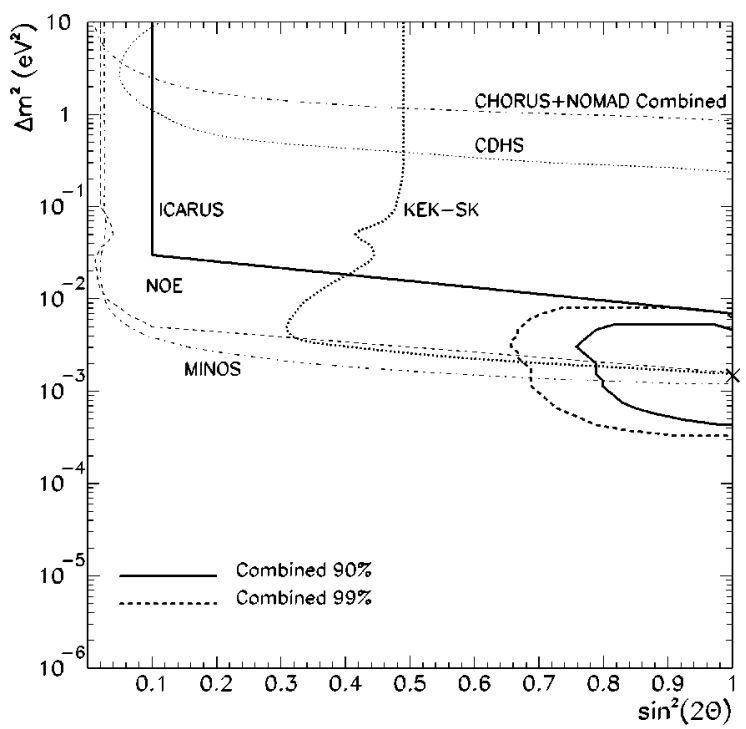

FIG. 14. The allowed $\nu_{\mu} \rightarrow \nu_{\tau}$ oscillation parameters for all experiments combined at 90 and $99 \%$ C.L. For a comparison we also display the presently excluded region from the accelerator experiments CDHSW and CHORUS + NOMAD and future long baseline experiments. The cross represents the best fit point. 
TABLE IV. Errors and correlations for both observed data and theory (MC) samples.

\begin{tabular}{lrrrrrr}
\hline \hline Experiment $(A)$ & \multicolumn{1}{c}{$\sigma_{\mu}^{\text {data }}$} & $\sigma_{e}^{\text {data }}(\%)$ & $\rho_{\mu e}^{\text {data }}(A, A)$ & $\sigma_{\mu}^{\text {theory }}$ & $\sigma_{e}^{\text {theory }}(\%)$ & $\rho_{\mu e}^{\text {theory }}(A, A)$ \\
\hline Fréjus & 10.5 & 17.9 & -0.021 & 31.7 & 31.9 & 0.951 \\
Kam sub-GeV & 7.1 & 7.0 & -0.081 & 31.7 & 31.8 & 0.975 \\
IMB & 8.9 & 7.5 & -0.374 & 36.1 & 36.1 & 0.947 \\
Nusex & 18.4 & 27.2 & -0.050 & 31.7 & 31.9 & 0.950 \\
Soudan2 & 13.5 & 11.0 & -0.168 & 30.8 & 31.1 & 0.960 \\
Super-Kam sub-GeV (unbinned) & 4.9 & 4.9 & -0.042 & 31.6 & 31.7 & 0.978 \\
Super-Kam sub-GeV bin1 & 9.4 & 8.3 & -0.013 & 31.7 & 31.8 & 0.936 \\
Super-Kam sub-GeV bin2 & 9.0 & 9.4 & -0.012 & 31.7 & 31.8 & 0.935 \\
Super-Kam sub-GeV bin3 & 9.0 & 8.4 & -0.013 & 31.7 & 31.8 & 0.936 \\
Super-Kam sub-GeV bin4 & 8.6 & 9.1 & -0.013 & 31.7 & 31.8 & 0.936 \\
Super-Kam sub-GeV bin5 & 8.3 & 9.8 & -0.012 & 31.7 & 31.8 & 0.935 \\
Kam multi-GeV (unbinned) & 9.6 & 11.0 & -0.038 & 31.7 & 32.0 & 0.965 \\
Kam multi-GeV bin1 & 24.0 & 22.2 & -0.008 & 31.8 & 33.9 & 0.840 \\
Kam multi-GeV bin2 & 22.8 & 23.3 & -0.008 & 31.9 & 32.9 & 0.869 \\
Kam multi-GeV bin3 & 18.2 & 19.1 & -0.012 & 31.8 & 32.6 & 0.889 \\
Kam multi-GeV bin4 & 18.8 & 22.8 & -0.009 & 31.9 & 32.8 & 0.878 \\
Kam multi-GeV bin5 & 17.2 & 33.6 & -0.007 & 31.9 & 33.8 & 0.838 \\
Super-Kam multi-GeV (unbinned) & 7.6 & 9.5 & -0.056 & 31.6 & 31.9 & 0.972 \\
Super-Kam multi-GeV bin1 & 17.9 & 21.9 & -0.010 & 31.7 & 32.4 & 0.911 \\
Super-Kam multi-GeV bin2 & 17.4 & 18.1 & -0.013 & 31.7 & 32.1 & 0.920 \\
Super-Kam multi-GeV bin3 & 12.4 & 18.9 & -0.017 & 31.7 & 32.1 & 0.923 \\
Super-Kam multi-GeV bin4 & 12.2 & 17.1 & -0.019 & 31.7 & 32.1 & 0.919 \\
Super-Kam multi-GeV bin5 & 12.7 & 19.8 & -0.016 & 31.7 & 32.4 & 0.909 \\
\hline \hline
\end{tabular}

CHORUS plus NOMAD combined limits [35], and the prospects for the future experiments being discussed at present can be appreciated in Fig. 14. One sees that the long-baseline experiments planned at KEK (K2K) [36], Fermilab (MINOS) [37] and CERN (NOE [38] and ICARUS [39]) fall short in sensitivity to probe the $\nu_{\mu}$ to $\nu_{\tau}$ oscillation parameters. This is in contrast with the situation in the pre-SuperKamiokande days. From this point of view experiments such as ICARUS and a re-design of experiments such as MINOS would be desired in order to enhance their sensitivity in testing the atmospheric neutrino anomaly.

\section{DISCUSSION AND CONCLUSIONS}

In this paper we have considered the impact of recent experimental results on atmospheric neutrinos from SuperKamiokande and Soudan2 as well as recent theoretical improvements in flux calculations and neutrino-nucleon cross sections on the determinations of atmospheric neutrino oscillation parameters, both for the $\nu_{\mu} \rightarrow \nu_{\tau}$ and $\nu_{\mu} \rightarrow \nu_{e}$ channels. The new Super-Kamiokande data cause a downwardshift in the $\left(\sin ^{2} 2 \theta, \Delta m^{2}\right)$ region, when compared with pre-SuperKamiokande results. We have also compared the results obtained in our fits of atmospheric neutrino data with previous results, as well as with the constraints following from laboratory searches for neutrino oscillations, both at accelerators and reactors. For example we have seen that the $\nu_{\mu} \rightarrow \nu_{e}$ oscillation hypothesis is barely consistent with the recent negative result of the $\mathrm{CHOOZ}$ reactor [13]. The sensitivity attained in atmospheric neutrino observations in the $\nu_{\mu}$ $\rightarrow \nu_{\tau}$ channel is also compared with those of accelerator neutrino oscillation searches, for example at CHORUS and NOMAD as well as at the future experiments being discussed at present. Especially interesting from our point of view are the long-baseline experiments planned at KEK (K2K), Fermilab (MINOS) and CERN (NOE, ICARUS). However, because of the lowering of the allowed $\left(\sin ^{2} 2 \theta, \Delta m^{2}\right)$ region, it is not clear whether a re-design is needed in some of these experiments, for example MINOS, in order to enhance their sensitivity in testing the atmospheric neutrino anomaly.

Note that, throughout this work we have assumed a rather generous error in the absolute fluxes of atmospheric neutrinos. We have investigated to some extent the effect a reduced error in the fluxes would have in the determination of neutrino oscillation parameters from the present atmospheric neutrino data. We have found no significant effect in the shape of an allowed region when we changed the assumed error in the fluxes from $30 \%$ to $20 \%$. However, we have noticed a somewhat significant effect of a more accurate ratio of muon-to-electron events. We have found, for example, for Super-Kamiokande, that when we decrease the error in the muon-to-electron-type event ratio from 5\% (10\%) for unbinned (binned) data to $3 \%(6 \%)$ the allowed region shrinks by about 10 to $15 \% \sin ^{2} 2 \theta$, close to 0.7 or so. There is hardly any effect in the $\Delta m^{2}$ range determination.

\section{ACKNOWLEDGMENTS}

This work was supported by Fundação de Amparo à Pesquisa do Estado de São Paulo (FAPESP), by DGICYT under 
Grant PB95-1077, CICYT under Grant AEN96-1718, and by the TMR network Grant ERBFMRXCT960090 of the European Union. H. Nunokawa was supported by a DGICYT grant; O. Peres by a grant from FAPESP. The research of T. $\mathrm{S}$. is supported in part by U.S. Department of Energy Contract DE-FG02-91ER40626. M. C. G-G is grateful to the Instituto de Física Teórica for its kind hospitality. We want to thank Takaaki Kajita, for providing us with the Kamioka efficiencies and for useful discussions, Kunio Inoue for providing us with the preliminary Super-Kamiokande acceptances, Hugh Gallagher, Yoichiro Suzuki, Chiaki Yanagisawa and Eligio Lisi, for useful correspondence and discussion and our colleagues Eulogio Oset and S. K. Singh, for useful discussions on the uncertainties in neutrino nucleus cross sections.

\section{APPENDIX: CORRELATION OF ERRORS}

Here we present the errors and correlations used in our analysis. In Table IV we display the errors and correlations $\rho_{\mu e}(A, A)$ for all the experiments. Data errors and correlations contain the experimental statistical errors as well as those due to misidentification as quoted by the experiments. In order to compute $\sigma_{e}^{\text {theory }}$ we take into account [27], the flux uncertainty, the MC statistical errors (which depend on the number of simulated MC events) as well as the cross sections uncertainties. The flux uncertainty is taken to be $30 \%$ whereas MC statistical errors are estimated under the assumption that the $\mu$ and $e$-like events follow a binomial distribution. Nuclear cross section uncertainties are taken to be $10 \%$ for all the experiments except for Soudan 2 because we used the values $7.5 \%$ and $6.4 \%$ for $e$-like and $\mu$-like events, respectively [10].

Data errors between different experiments are assumed to be uncorrelated,

$$
\begin{aligned}
& \rho_{\alpha \alpha}^{\text {data }}(A, A)=1(\alpha=e, \mu) \text { for all } A, \\
& \rho_{\alpha \beta}^{\text {data }}(A, B)=0(\alpha, \beta=e, \mu) \text { if } A \neq B,
\end{aligned}
$$

while the theory correlations between different experiments (i.e., for $A \neq B$ ) are obtained as follows:

$$
\begin{aligned}
& \rho_{\alpha \beta}^{\text {theory }}(A, B)=\rho_{\alpha \beta}^{\text {flux }} \times \frac{\sigma_{\alpha}^{\text {flux }} \sigma_{\beta}^{\text {flux }}}{\sigma_{\alpha}^{\text {theory }}(A) \sigma_{\alpha}^{\text {theory }}(B)} \quad \text { if } \alpha \neq \beta, \\
& \rho_{\alpha \beta}^{\text {theory }}(A, B)=\rho_{\alpha \beta}^{\text {flux }} \times \frac{\sigma_{\alpha}^{\text {flux }} \sigma_{\beta}^{\text {flux }}}{\sigma_{\alpha}^{\text {theory }}(A) \sigma_{\beta}^{\text {theory }}(B)} \quad \text { if } \alpha=\beta,
\end{aligned}
$$

where $\sigma_{e}^{f l u x}=\sigma_{\mu}^{f l u x}=30 \%$ and $\rho_{\alpha \beta}^{\text {flux }}=1.000$ for $\alpha=\beta$. For $\alpha \neq \beta$, we use $\rho_{\alpha \beta}^{\text {flux }}=0.986(0.944)$ as determined from the relation

$$
\left(\sigma_{\mu / e}^{f l u x}\right)^{2}=\left(\sigma_{\mu}^{f l u x}\right)^{2}+\left(\sigma_{e}^{f l u x}\right)^{2}-2 \rho_{\mu e}\left(\sigma_{\mu}^{f l u x}\right)\left(\sigma_{e}^{f l u x}\right)
$$

after imposing that the uncertainty in the flavor ratio $\sigma_{\mu / e}^{\text {flux }}$ $=5 \%(10 \%)$ for unbinned (binned) case [27]. Furthermore we assume that there is no correlation between the sub-GeV and multi-GeV data.

We note that for both sub-GeV and multi-GeV data, in general, $\rho_{\alpha \beta}^{\text {theory }}(A, B)$ is not symmetric under the exchange of the flavor labels $\alpha$ and $\beta$ or the experimental labels, i.e.,

$$
\begin{aligned}
& \rho_{\alpha \beta}^{\text {theory }}(A, B) \neq \rho_{\beta \alpha}^{\text {theory }}(A, B) \text { if } \alpha \neq \beta, \\
& \rho_{\alpha \beta}^{\text {theory }}(A, B) \neq \rho_{\alpha \beta}^{\text {theory }}(B, A) \text { if } A \neq B,
\end{aligned}
$$

but it is symmetric under simultaneous exchange of both kinds of labels $\alpha, \beta$ and $A, B$,

$$
\rho_{\alpha \beta}^{\text {theory }}(A, B)=\rho_{\beta \alpha}^{\text {theory }}(B, A) .
$$

[1] For reviews, see, e.g., T. K. Gaisser, in Neutrino '96, Proceedings of the 17th International Conference on Neutrino Physics and Astrophysics, Helsinki, Finland, edited by K. Enquist, K. Huitu, and J. Maalampi (World Scientific, Singapore, 1997), p. 211; T. K. Gaisser, F. Halzen, and T. Stanev, Phys. Rep. 258, 174 (1995); T. Stanev, in Proceedings in TAUP '95, Toledo, Spain, edited by A. Morales, J. Morales, and J. A. Villar [Nucl. Phys. B (Proc. Suppl.) 48, 165 (1996)]; T. Kajita, in Physics and Astrophysics of Neutrinos, edited by M. Fukugita and A. Suzuki (Springer-Verlag, Tokyo, 1994), p. 559; E. Kh. Akhmedov, in Cosmological Dark Matter, Proceedings of the International School on Cosmological Dark Matter, Valencia, Spain, edited A. Perez and J. W. F. Valle (World Scientific, Singapore, 1994), p. 131.

[2] Stanev [1].

[3] B. Barish, in Proceedings of Int. Workshop on Elementary Particle Physics: Present and Future, edited by A. Ferrer and J. W. F. Valle (World Scientific, Singapore, 1996), p. 400.

[4] Kamiokande Collaboration, H. S. Hirata et al., Phys. Lett. B
205, 416 (1988); 280, 146 (1992); Kamiokande Collaboration, K. Kaneyuki et al., in Perspectives in Neutrinos, Atomic Physics and Gravitation, Proceedings of the XIII Moriond Workshop, edited by J. Tran Thanh Van et al. (Editions Frontieres, Villars sur Ollon, Switzerland, 1993), p. 211.

[5] Kamiokande Collaboration, Y. Fukuda et al., Phys. Lett. B 335, 237 (1994).

[6] IMB Collaboration, D. Casper et al., Phys. Rev. Lett. 66, 2561 (1991); R. Becker-Szendy et al., Phys. Rev. D 46, 3720 (1992); IMB Collaboration, D. Keilczewska et al., Perspectives in Neutrinos, Atomic Physics and Gravitation, Proceedings of the XIII Moriond Workshop, edited by J. Tran Thanh Van et al. (Editions Frontieres, Villars sur Ollon, Switzerland, 1993), p. 219.

[7] Fréjus Collaboration, Ch. Berger et al., Phys. Lett. B 227, 489 (1989).

[8] NUSEX Collaboration, M. Aglietta et al., Europhys. Lett. 8, 611 (1989).

[9] Super-Kamiokande Collaboration, K. Nakamura, in XVI Inter- 
national Workshop on Weak Interactions and Neutrinos, Capri, Italy, 1997, edited by G. Fiorilo et al. [Nucl. Phys. B (Proc. Suppl.) 66, 282 (1998)]; Super-Kamiokande Collaboration, E. Kearns et al., talk given at TAUP97, Laboratori Nazionali del Gran Sasso, Assergi, Italy, 1997, hep-ex/9803007.

[10] Soudan Collaboration, W. W. M Allison et al., Phys. Lett. B 391, 491 (1997); Soudan Collaboration, Hugh Gallagher et al., in XVI International Workshop on Weak Interactions and Neutrinos [9], p. 290; Soudan Collaboration, T. Kafka et al. talk given at TAU97, Laboratori Nazionali del Gran Sasso, Assergi, Italy, 1997, hep-ph/9712281.

[11] V. Agrawal et al., Phys. Rev. D 53, 1314 (1996); T. K. Gaisser and T. Stanev, in Proceedings of the 24th ICRC, edited by B. D. Piazzoli et al., Rome, 1995, Vol. 1, p. 694.

[12] L. V. Volkova, Sov. J. Nucl. Phys. 31, 784 (1980).

[13] CHOOZ Collaboration, M. Apollonio et al., Phys. Lett. B 420, 397 (1998).

[14] G. Barr, T. K. Gaisser, and T. Stanev, Phys. Rev. D 39, 3532 (1989); 38, 85 (1988).

[15] P. Lipari and T. Stanev, in Proceedings of the 24th ICRC [11], Vol. 1.

[16] M. Honda, T. Kajita, K. Kasahara, and S. Midorikawa, Phys. Rev. D 52, 4985 (1995); M. Honda, K. Kasahara, K. Hidaka, and S. Midorikawa, Phys. Lett. B 248, 193 (1990).

[17] T. K. Gaisser et al., Phys. Rev. D 54, 5578 (1996).

[18] E. V. Bugaev and V. A. Naumov, Phys. Lett. B 232, 391 (1989)

[19] M. Circella et al., in Proceedings of the 25th ICRC, Durban, 1997, Vol. 7, p. 117.

[20] T. K. Gaisser and T. Stanev, Phys. Rev. D 57, 1977 (1998).

[21] P. Lipari, M. Lusignoli, and F. Sartogo, Phys. Rev. Lett. 74, 4384 (1995).

[22] C. L. Smith, Phys. Rep., Phys. Lett. 3C, 261 (1972).

[23] G. L. Fogli and G. Nardulli, Nucl. Phys. B160, 116 (1979); M. Nakahata et al., J. Phys. Soc. Jpn. 55, 3786 (1986).

[24] G. L. Fogli, E. Lisi, and D. Montanino, Phys. Rev. D 49, 3626
(1994); Astropart. Phys. 4, 177 (1995); G. L. Fogli, E. Lisi, D. Montanino, and G. Scioscia, ibid. 55, 4385 (1997).

[25] T. Kajita (private communication); see also Kajita [1].

[26] K. Inoue (private communication).

[27] G. L. Fogli and E. Lisi, Phys. Rev. D 52, 2775 (1995).

[28] S. P. Mikheyev and A. Yu. Smirnov, Yad. Fiz. 42, 1441 (1985); L. Wolfenstein, Phys. Rev. D 17, 2369 (1985).

[29] E. Lisi and D. Montanino, Phys. Rev. D 56, 1792 (1997).

[30] G. S. Vidyakin et al., JETP Lett. 59, 390 (1994).

[31] B. Achkar et al., Nucl. Phys. B424, 503 (1995).

[32] CDHSW Collaboration, F. Dydak et al., Phys. Lett. 134B, 281 (1984); E776 Collaboration, L. Borodvsky et al., Phys. Rev. Lett. 68, 274 (1992); E531 Collaboration, N. Ushida et al., Phys. Rev. Lett. 57, 2897 (1986).

[33] CHORUS Collaboration, N. Armenise et al., Report No. CERN-SPSC/90-42 (1990).

[34] NOMAD Collaboration, P. Astier et al., Report No. CERNSPSLC/91-21 (1991); Report No. CERN-SPSLC/91-48 (1991); Report No. SPSLC/P261 Add. 1 (1991).

[35] L. Di Lella, talk given at TAUP97, Laboratori Nazionali del Gran Sasso, Assergi, Italy, 1997.

[36] KEK-SK Collaboration, C. Yanagisawa et al., talk given at International Workshop on Physics Beyond The Standard Model: from Theory to Experiment, Valencia, Spain, 1997, to appear in the proceedings, edited by I. Antoniadis, L. Ibanez and J. W. F. Valle (World Scientific, Singapore, 1998).

[37] MINOS Collaboration, Dave Ayres et al., NuMI-L-63 MINOS proposal; http://www.hep.anl.gov/NDK/HyperText/numi.html

[38] NOE Collaboration, M. Ambrosio et al., Nucl. Instrum. Methods Phys. Res. A 363, 604 (1995), http://www.na.infn.it/ SubNucl/accel/noe/noe.html

[39] ICARUS Collaboration, http://www.aquila.infn.it/icarus/; F. Cavanna et al., "A first 600 ton ICARUS detector installed at the Gran Sasso Laboratory," addendum to Proposal by the ICARUS Collaboration, Report No. LNGS-95/10 (1995). 